\title{
Coherent Transport through an interacting double quantum dot: Beyond sequential tunneling
}

\author{
Jonas Nyvold Pedersen, Benny Lassen * and Andreas Wacker \\ Mathematical Physics, Physics Department, Lund University, Box 118, 22100 Lund, Sweden \\ Matthias H. Hettler \\ Forschungszentrum Karlsruhe, Institut für Nanotechnologie, Postfach 3640, 76021 Karlsruhe, Germany
}

(Dated: February 6, 2008)

\begin{abstract}
Various causes for negative differential conductance in transport through an interacting double quantum dot are investigated. Particular focus is given to the interplay between the renormalization of the energy levels due to the coupling to the leads and the decoherence of the states. The calculations are performed within a basis of many-particle eigenstates and we consider the dynamics given by the von Neumann-equation taking into account also processes beyond sequential tunneling. A systematic comparison between the levels of approximation and also with different formalisms is performed. It is found that the current is qualitatively well described by sequential processes as long as the temperature is larger than the level broadening induced by the contacts.
\end{abstract}

\section{INTRODUCTION}

The field of electronic transport through metallic and semi-conducting quantum dots has been a topic of intense research for over a decade. While in the early experiments the main focus was on charging effects leading to the phenomenon known as 'Coulomb blockade',, and later on the Kondo effect in single quantum dots, $\underset{2}{2}$ in recent years the attention has shifted to more elaborate systems such as double quantum dot systems $, 3,4,5,6,7,8,9$ In addition, the study of the electronic spectrum of quantum dots (excited states) was possible as the physical size of quantum dots could be further reduced by improved lithographical methods as well as the rise of new materials such as nanotubes and semi-conducting nanowires. 10

Already in the '90s, there were experiments ${ }^{11}$ on single quantum dots displaying non-monotonous currentvoltage characteristics, for which the current decreases with increasing bias, leading to a negative differential conductance (NDC). Within the orthodox theory of sequential tunneling, such effects were explained by the presence of excited states which were more weakly coupled to the leads than the ground states (for a given charge on the dot). The reason for a state dependent coupling could be either due to spin (Clebsch-Gordon coefficients $)^{12}$ or the different orbital wave-functions of the various states. 13,14 A bias voltage dependence of the lead-dot tunnel coupling can lead to a weak NDC effect, see, e.g., Ref. 15.

For a double quantum dot (DQD) system with the two quantum dots in series, very sharp current peaks (and corresponding NDC) was observed in experiments where the inter-dot coupling $\Omega$ was weak ${ }^{3}$ Depending on whether two levels on different dots are 'aligned' or not, the current will be high (in alignment) or low (off alignment). As the quantum levels in the different dots are shifted differently by the applied bias voltage (depending on the various capacitances of the system) the alignment condition is fulfilled at certain small ranges of the bias voltage, leading to the current peaks.

In this paper we focus on quantum transport through a DQD. This system offers the possibility to study the interplay between the coherent quantum mechanical oscillation inside the DQD and the influence of the coupling to leads. Especially, we are interested in the negative differential conductance caused by this interplay (rather than the well-known sources discussed above). In the regime where the inter-dot tunneling coupling dominates over the coupling to leads, it was found in Ref. 16 that NDC only occurs if the spatial symmetry of the system is broken, e.g. due to asymmetric coupling to leads or detuning of the bare level energies $\stackrel{40}{ }$ In another recent paper, B. Wunsch et al. [17] investigated the transport in the opposite limit, where the inter-dot coupling is weak and with a small detuning of the bare level energies, i.e. for an asymmetric system. They found that NDC can be caused by a level renormalization due to the coupling to leads. Also in the weak inter-dot coupling regime, Djuric et al. [18] found NDC even for symmetric systems for certain ratios between the inter-dot tunneling coupling and the coupling to leads. The effect was explained in terms of decoherence due to the coupling to leads, which depended on the occupation of the dot. In this article we show how these effects relate to each other.

In the above mentioned works, the current was only calculated to lowest (first) order in the lead-dot tunnel coupling, so strictly speaking, the results are only valid in the sequential tunneling limit. However, for the issues addressed above, the coupling to the contacts strongly influences not only the occupations but also affects the nature of the transport, especially its quantum-mechanical coherence. Thus it is not a priori clear, if the first-order approach is appropriate, even if the temperature is higher than the energy scale (line-width) due to the coupling to the leads. Below, we investigate the current to higher order in the lead-dot coupling by applying the method described in Ref. 19 and compare with first order results. For both cases we find qualitative agreement if the tem- 
perature is higher or comparable to the line-width due to coupling to the leads, but we also discuss the behavior for lower temperatures, where the first order approach becomes unreliable.

The paper is organized as follows: in section \we present in detail the DQD model system we consider. Section [III discusses in brief the transport models we are using to obtain the transport results (details are presented in the appendix). The potential sources of NDC behavior are discussed in detail in section IV Finally we summarize our findings in section $\mathrm{V}$

\section{THE MODEL SYSTEM}

We consider a double quantum dot system, where the spin degree of freedom has been omitted in order to simplify the analysis (in subsection IVE the double spin case is briefly addressed). In a single-particle basis the Hamiltonian for the system reads

$$
\begin{aligned}
& H=E_{\alpha} d_{\alpha}^{\dagger} d_{\alpha}+E_{\beta} d_{\beta}^{\dagger} d_{\beta}+U d_{\alpha}^{\dagger} d_{\alpha} d_{\beta}^{\dagger} d_{\beta}+\left(\Omega d_{\beta}^{\dagger} d_{\alpha}+h . c\right) \\
& +\sum_{k \ell} E_{k \ell} c_{k \ell}^{\dagger} c_{k \ell}+\sum_{k}\left(t_{k L} d_{\alpha}^{\dagger} c_{k L}+t_{k R} d_{\beta}^{\dagger} c_{k R}+\text { h.c. }\right)
\end{aligned}
$$

where the first line describes the isolated quantum dot system with $U$ being the Coulomb energy for occupying both dots, $\Omega$ the inter-dot tunneling coupling and with $\alpha / \beta$ denoting the left/right dot. The first term in the second line accounts for the leads with index $\ell=L / R$ for the left/right lead and levels counted by $k$. The last term is the lead-dot tunneling coupling. We parameterize the lead-dot coupling parameters $t_{k \ell}$ by $\Gamma_{\ell}(E)=2 \pi \sum_{k}\left|t_{k \ell}\right|^{2} \delta\left(E-E_{k \ell}\right)$. Here we use the constant value $\Gamma_{\ell}$ for $|E| \leq 0.95 W$ and assume $\Gamma_{\ell}(E)=0$ for $|E|>W$. For $0.95 W<|E|<W$ we interpolate with an elliptic behavior in order to avoid discontinuities. Furthermore, we define $\Gamma=\Gamma_{L}+\Gamma_{R}$. The bias voltage $V_{\text {bias }}$ is applied symmetrically to the electrochemical potentials of both leads, $\mu_{L}=-\mu_{R}=e V_{\text {bias }} / 2$, where $e$ is the positive elementary charge.

Throughout this paper we include the Coulomb interaction by considering a basis of many-particle states for the isolated DQD, which allows for a consistent description of many-particle effects, see also Ref. 20 and references given therein. Thus, we diagonalize the first line of the Hamiltonian from Eq. (11) and find the eigenstates and the corresponding energies. $E_{0}=0$ is the energy of the empty state, $E_{1}=\rho-\sqrt{\Delta^{2}+4 \Omega^{2}} / 2$ and $E_{2}=\rho+\sqrt{\Delta^{2}+4 \Omega^{2}} / 2$ the energies of the single occupied states, and $E_{d}=E_{\alpha}+E_{\beta}+U$ the energy of the double occupied state, where $\Delta=E_{\alpha}-E_{\beta}$ and $\rho=\left(E_{\alpha}+E_{\beta}\right) / 2$. The states with energies $E_{1} / E_{2}$ are referred to as the bonding/anti-bonding state.

Depending on the occupation of the dot states different transport regimes can be defined. Current through the
DQD is effectively blocked if no one-particle excitation lies in the bias window between the Fermi levels of both contacts. This is known as the Coulomb blockade regime. Therefore, as the bias is increased, a current can flow through the structure whenever a one-particle excitation becomes energetically allowed, leading to a step feature in the current and a corresponding peak in the differential conductance. Having 4 such possible excitations $(0 \leftrightarrow$ $1,2$, and $1,2 \leftrightarrow d)$, at most four steps can be observed in the $I V$-curve. Further steps can be seen, if spin is considered as well $\stackrel{21}{2}$

The quantum rate equations from Refs. 22, 23,24 are valid in the high-bias limit, i.e. if the energy difference between the chemical potentials in the contacts and the excitations exceeds both the level broadenings $\Gamma_{\ell}$ and the temperature. If only one-particle states are within the bias window, but double occupation is forbidden, i.e., $\left(E_{d}-E_{1}\right),\left(E_{d}-E_{2}\right) \gg \mu_{L}, \mu_{R}$, these equations provide the plateau current

$$
I_{1}=\frac{e}{h} \frac{\Omega^{2} \Gamma_{R}}{\Omega^{2}\left(2+\Gamma_{R} / \Gamma_{L}\right)+\left(\Gamma_{R} / 2\right)^{2}+\Delta^{2}} .
$$

If, in contrast, all excitations are within the bias window, one obtains

$$
I_{2}=\frac{e}{h} \frac{\Gamma_{L} \Gamma_{R} \Gamma \Omega^{2}}{\left(4 \Omega^{2}+\Gamma_{L} \Gamma_{R}\right)(\Gamma / 2)^{2}+\Delta^{2} \Gamma_{L} \Gamma_{R}},
$$

These values will be compared to our calculations in the subsequent sections.

\section{THE VON NEUMANN APPROACH}

Our calculations are based on the von Neumann equation for the density matrix, as described in detail in Ref. 19. The key idea is to use a set of many-particle states labeled $|a\rangle,|b\rangle, \ldots$, with energies $E_{a}, E_{b}, \ldots$, respectively, which diagonalize the Hamiltonian of the system, including the many-particle interaction. (In our case these are the states $|0\rangle,|1\rangle,|2\rangle,|d\rangle$ introduced in section [II.) Transport occurs by tunneling of electrons with a quantum number $k$ from a lead $\ell$ into the system, while the state is changed from an $N$-particle state $|a\rangle$ to an $N+1$-particle state $|b\rangle$. The corresponding matrix element is $T_{b a}(k \ell)$. In Ref. 19 the full correlations of up to two particles entering and leaving the system was taken into account and this approach will be referred to as the second order von Neumann approach $(2 \mathrm{vN})$ in the following. In addition, we apply the same concept restricting to single electron processes, which we call the first order von Neumann approach $(1 \mathrm{vN})$. The resulting equations for the $1 \mathrm{vN}$ approach are given in App. $\mathrm{A}$ Both the first and the second order approaches include the nondiagonal elements of the density matrix, which allows us to consider the regime of both weak and strong inter-dot coupling $\Omega$. This is demonstrated in App. B where our method is also compared with other approaches. The 
$1 \mathrm{vN}$ approach neglects level broadening effects (of order $\Gamma$ ) and is thus expected to be valid only for $k_{B} T \gg \Gamma$ or in the high-bias limit. In contrast, the $2 \mathrm{vN}$ approach is able to reproduce such effects and gives good results above the Kondo temperature $\frac{19}{\underline{1}}$ Due to the self-consistency, the $2 \mathrm{vN}$ approach contains in addition to two-particle correlations also a subset of higher order correlations. In App. C] we show that the result for the Anderson model is identical with the corresponding result obtained from the Real-Time diagrammatic approach in resonant tunneling approximation $:^{25,26}$

The $1 \mathrm{vN}$ approach contains sums of the form $\sum_{k} T_{b a} T_{b^{\prime} a^{\prime}}^{*} /\left(E_{k}-E_{b^{\prime}}+E_{a}-\mathrm{i} 0^{+}\right)$(see App. A). Decomposing

$$
\begin{aligned}
\frac{1}{E_{k}-E_{b^{\prime}}+E_{a}-\mathrm{i} 0^{+}} & =\mathcal{P}\left\{\frac{1}{E_{k}-E_{b^{\prime}}+E_{a}}\right\} \\
& -\mathrm{i} \pi \delta\left(E_{k}-E_{b^{\prime}}+E_{a}\right),
\end{aligned}
$$

the imaginary part can be related to electronic transition rates, while the real part acts as an effective renormalization of the transition energies between different manyparticle states. In some calculations we will neglect all terms resulting from these real parts (we denote this by "no real parts") in order to demonstrate their relevance.

\section{SOURCES FOR NDC BEHAVIOR}

In a real experimental double-dot structure, the applied source-drain bias $V_{\text {bias }}$ does not only determine the electrochemical potentials in the leads, but it will also shift the dot level energies by polarization. The amount of these shifts depends on the details of the various dot capacitances and can be taken into account by lever arm factors $\lambda_{\alpha}, \lambda_{\beta}$ for the respective dot levels ${ }^{3}$. In addition, if gates are present, the gate voltages $V_{\text {gate }}^{\alpha / \beta}$ can also shift the respective dot levels with efficiency factors $\eta_{\alpha}, \eta_{\beta}$. Therefore, the voltage dependence of the dot level energies can be written as

$$
\begin{aligned}
& E_{\alpha}=E_{\alpha}^{0}+\lambda_{\alpha} \frac{e V_{\text {bias }}}{2}-\eta_{\alpha} e V_{\text {gate }}^{\alpha}, \\
& E_{\beta}=E_{\beta}^{0}-\lambda_{\beta} \frac{e V_{\text {bias }}}{2}-\eta_{\beta} e V_{\text {gate }}^{\beta}
\end{aligned}
$$

with $E_{i}^{0}$ being the equilibrium level of the energies. This allows for an independent control of $V_{\text {bias }}$, the level difference (detuning) $\Delta=E_{\alpha}-E_{\beta}$, and the average level $\rho=\left(E_{\alpha}+E_{\beta}\right) / 2$. In the following we set $\rho=0$, meaning that the dot states are at equal energetic distance from the equilibrium Fermi level. In Fig. [1 we show the current calculated with the $1 \mathrm{vN}$ approach as a function of $V_{\text {bias }}$ and $\Delta$ for different inter-dot coupling strengths $\Omega$.

In a real experiment, the current voltage characteristic corresponds typically to a line in the $\left(V_{\mathrm{bias}}, \Delta\right)$-plane. With zero gate voltages, one has

$$
\Delta=E_{\alpha}^{0}-E_{\beta}^{0}+\left(\lambda_{\alpha}+\lambda_{\beta}\right) \frac{e V_{\text {bias }}}{2},
$$

(a) $\Omega=\Gamma / 10, k_{B} T=\Gamma$

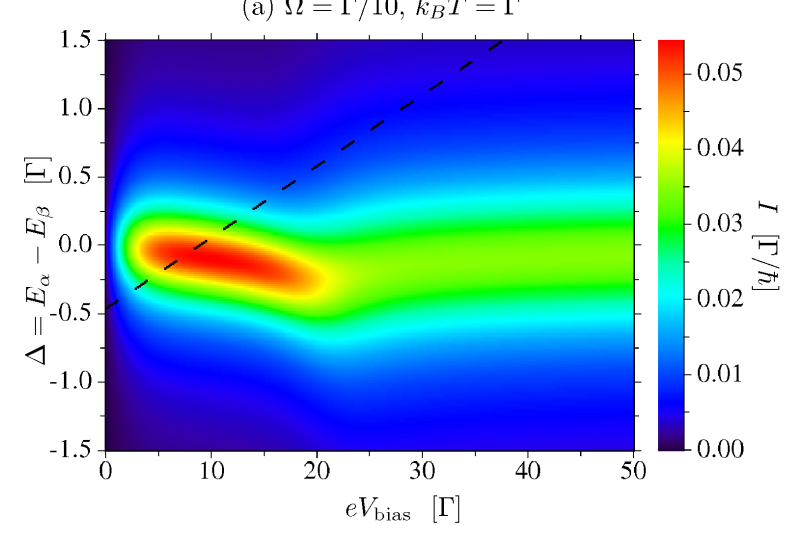

(b) $\Omega=\Gamma / 4, k_{B} T=\Gamma$

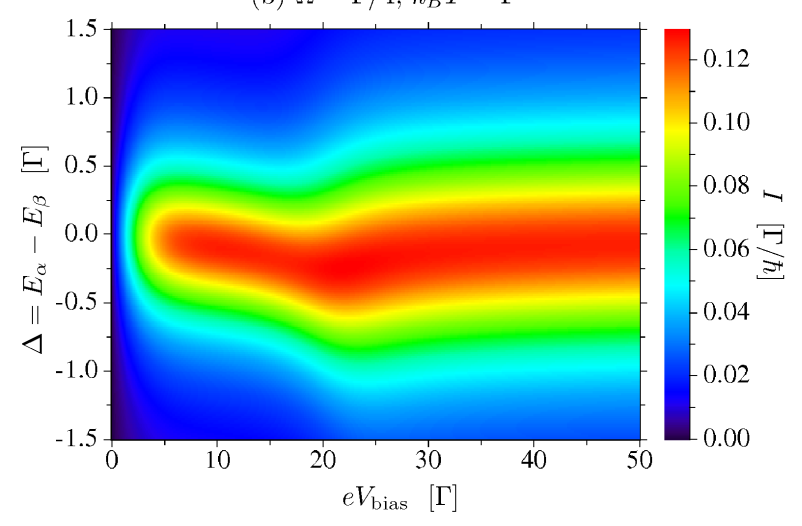

(c) $\Omega=2 \Gamma, k_{B} T=\Gamma$

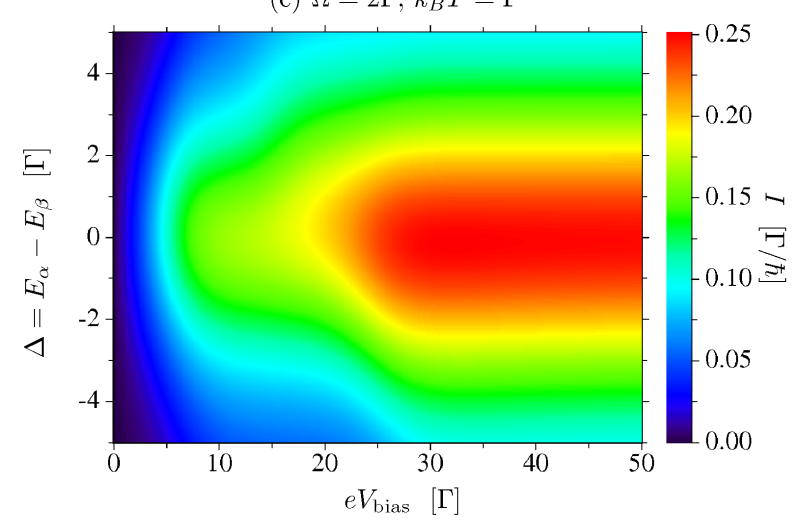

FIG. 1: Current versus bias voltage and detuning $\Delta=E_{\alpha}-$ $E_{\beta}$ for (a) $\Omega=\Gamma / 10$, (b) $\Omega=\Gamma / 4$, and (c) $\Omega=2 \Gamma$ using the first order approach $(1 \mathrm{vN})$ including the real parts. The other parameters are $E_{\alpha}+E_{\beta}=0, \Gamma_{L}=\Gamma_{R}=\Gamma / 2, k_{B} T=\Gamma$, $U=10 \Gamma$ and $W=35 \Gamma$.

so one follows a straight line with positive slope, see, e.g., the dashed line in Fig. 1(a). For a sufficiently large slope $\left(\lambda_{\alpha}+\lambda_{\beta}\right) / 2$, we observe first an increase of current for low bias, and then a decrease of the current as the levels move out of resonance with increasing $\Delta$. This is the standard NDC effect induced by electrostatic polarization.

In addition to the above 'trivial' effect we can identify two further scenarios for NDC for a fixed detuning $\Delta^{41}$, 

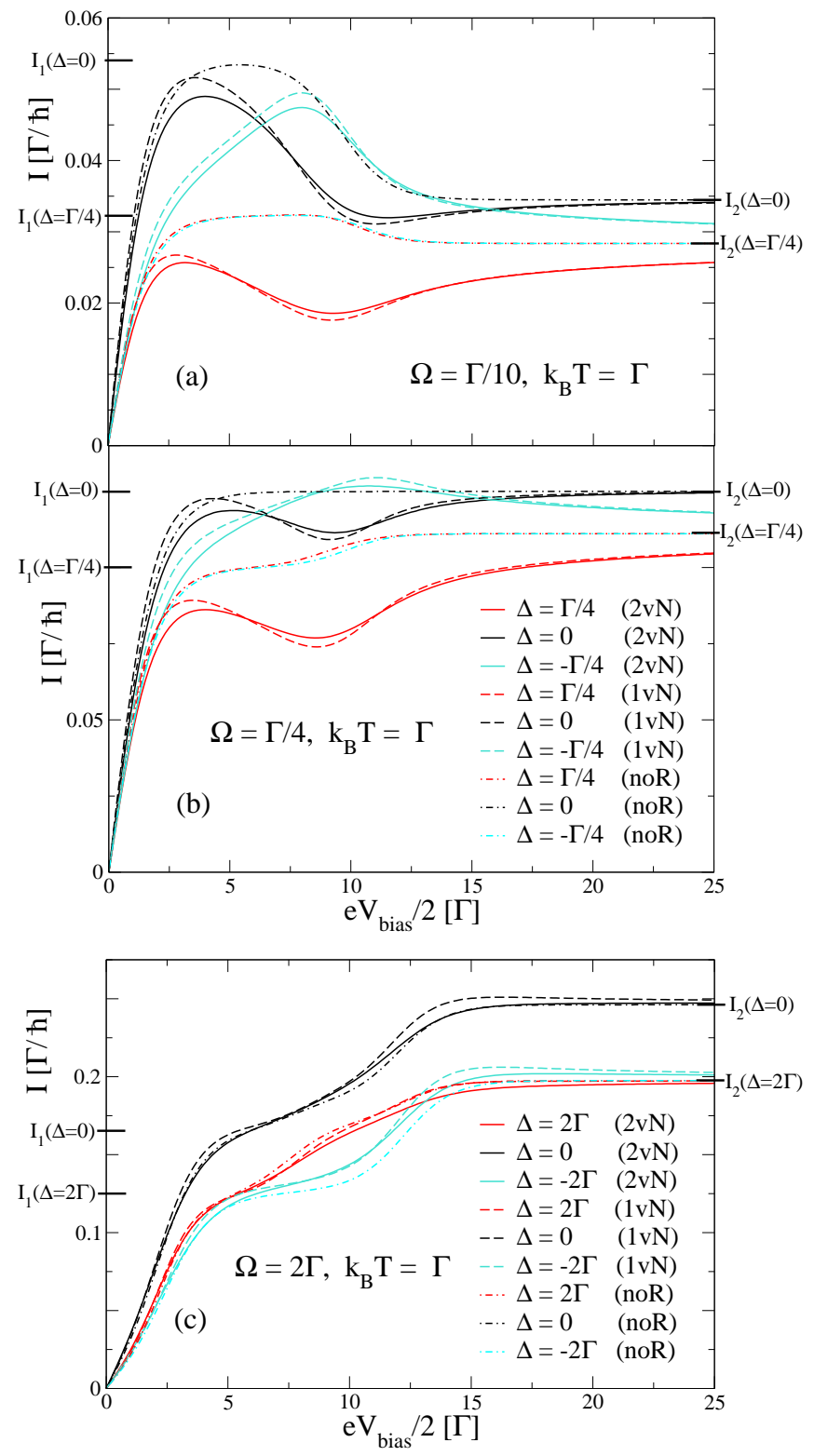

FIG. 2: Current versus bias voltage for different detunings $\Delta$. All other values like in Fig. 1 The dashed lines are the $1 \mathrm{vN}$ results including the real parts, the dashed-dotted lines are the $1 \mathrm{vN}$ results without the real parts (noR), and the full lines are the $2 \mathrm{vN}$ results. The values from Eqs. (2)3) for $\Delta=0$ and $\Delta= \pm \Gamma / 4$ or $\Delta= \pm 2 \Gamma$ (which do not depend on the sign of $\Delta$ ) are shown on the $y$-axis. We only show positive bias here, as the negative bias result corresponds to the results with the opposite sign of $\Delta$ for the symmetric coupling to contacts considered here.

which will be discussed in detail below. Firstly, we notice that for small and intermediate $\Omega$ [Figs. 1 (a,b)] the current peak (red region) is shifted to negative $\Delta$ with increasing bias $0<e V_{\text {bias }}<20 \Gamma=2 U$. Thus, the current drops with $V_{\text {bias }}$ if $\Delta \gtrsim 0$ is kept constant, see also the dashed lines in Fig. 2(a,b). A more detailed analysis is given in Sec. IVB.
Secondly, the height of the current peak (at fixed bias) drops when the bias voltage exceeds $2 U=20 \Gamma$ in Fig 1(a) where the inter-dot tunneling coupling is much weaker than the coupling to leads. This provides NDC around $\Delta \approx 0$ as discussed in detail in Sec. IVD, With increasing inter-dot coupling this effect vanishes as seen in Figs. 1(bc).

\section{A. First order versus second order}

In Fig. 2 we provide a systematic comparison between the $1 \mathrm{vN}$ approach (dashed line) and the $2 \mathrm{vN}$ approach (full line) for different values of the detuning $\Delta$, corresponding to cuts along horizontal lines in Fig. 1. We find that both approaches are in good qualitative agreement both for large and small values of the interdot coupling $\Omega$ at the considered temperature. This shows that the $1 \mathrm{vN}$ approach works well even for the moderate temperature $k_{B} T=\Gamma_{L}+\Gamma_{R}$. We observe small discrepancies close to current steps, where the broadening is underestimated due to the neglect of line-width broadening in the $1 \mathrm{vN}$ approach. As expected, these discrepancies are strongly enhanced if the temperature drops below the level broadening as shown in Fig. B(b) for $k_{B} T=\left(\Gamma_{L}+\Gamma_{R}\right) / 5$.

\section{B. NDC due to level renormalization}

Let us now focus towards the bias range $e V_{\text {bias }}<2 U$, where the double occupied state does not yet contribute to the current. In this regime we observe a significant shift of the current peak from its naively expected position at $\Delta=0$ for small and intermediate $\Omega$ [Figs. 1(a,b)].

In Ref. 17 Wunsch et al. considered a DQD including spin. They calculated the transport using a firstorder diagrammatic real-time transport approach (see e.g. Refs. 25, 26), restricting themselves to the limit of small inter-dot coupling $\Omega \ll \Gamma$, where electronic states localized on the single dots form an appropriate basis. For positive $\Delta$ they observe pronounced NDC similar to Fig. 2(a,b). This was explained in the following way: The energy levels $E_{\alpha}, E_{\beta}$ are renormalized due to the couplings to the contacts. For finite $U$ this renormalization is strongest if the levels are close to the chemical potential. Now, the localized states used in Ref. 17 couple mostly to the nearest lead and thus the renormalization differs for both levels at finite bias. This provides a bias-dependent renormalized $\Delta_{\text {eff }}$ and the maximum of the current occurs at $\Delta_{\text {eff }}=0$ rather than $\Delta=0$. This effect does not occur for $U=0$ because in this case the renormalization does not depend on the location of the chemical potential (this becomes obvious in a Green function treatment providing the exact result for $U=0$ ).

Our results in Figs. 1(a,b) are in full agreement with these findings. In particular, the shift of the current peak position can be directly attributed to a bias-dependent renormalization $\Delta \rightarrow \Delta_{\text {eff }}$, where $\Delta_{\text {eff }}>\Delta$ for positive 
(a) $\Omega=\Gamma / 10, k_{B} T=\Gamma / 5$
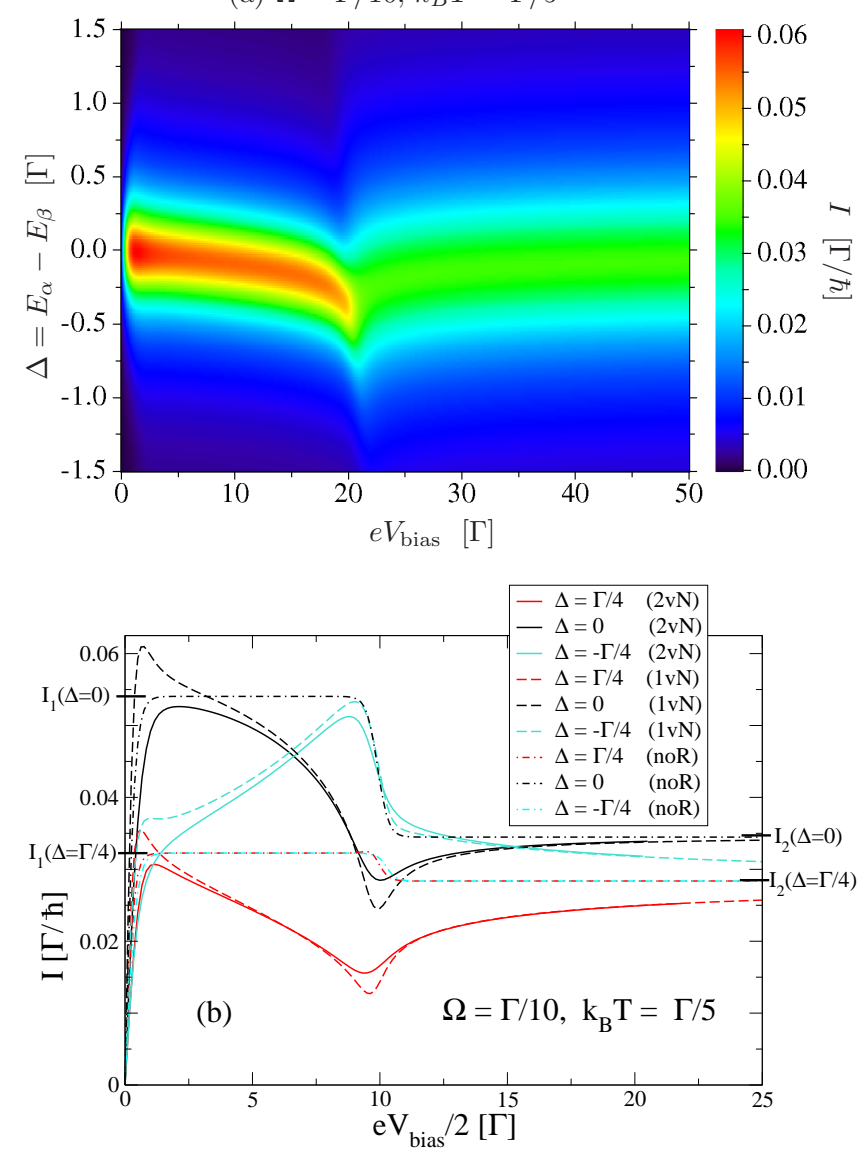

FIG. 3: Results for low temperature $k_{B} T=\Gamma / 5$ and $\Omega=$ $\Gamma / 10$. All other parameters as Figs. 112 Upper panel: Results of the $1 \mathrm{vN}$ approach. Lower panel: Comparison of $1 \mathrm{vN}, 2 \mathrm{vN}$ and the $1 \mathrm{vN}$ without real parts (noR) approaches. The $1 \mathrm{vN}$ strongly exaggerates the features at the current steps for this low temperature in comparison to the $2 \mathrm{vN}$ approach. The $1 \mathrm{vN}$ without real parts is qualitatively incorrect.

bias. This renormalization is also reflected in the magnitude of the current: For $e V_{\text {bias }}<2 U$, Eq. (2) suggests the plateau current $I_{1}$. In contrast, no such plateau is seen in Fig. 2( $(a, b)$ for small and intermediate tunnel coupling. Furthermore, the calculated currents are smaller than the respective value of $I_{1}(|\Delta|)$ for $\Delta=0$ and $\Delta=\Gamma / 4$, while they exceed $I_{1}(|\Delta|)$ for $\Delta=-\Gamma / 4$. This can be fully attributed to a bias-dependent renormalized $\Delta_{\text {eff }}>\Delta$ which should be used in Eq. (2).

Fig.1(c) shows that the effect vanishes for strong interdot coupling $\Omega>\Gamma$, a regime where the approach of Ref. 17 fails. In addition, the quantitative agreement between the $1 \mathrm{vN}$ and $2 \mathrm{vN}$ approach shows that the shift of the resonance condition is not an artefact of a first order tunneling approach (such as $1 \mathrm{vN}$ or the approach used in Ref. 17) but persist even if higher order tunneling processes are taken into account, which, e.g., cause linewidth broadening.

\section{Symmetry with respect to the sign of $\Delta$}

The quantum rate equation results Eqs. (213) only depend on the absolute value $|\Delta|$. In contrast, Figs. 112 exhibit a strong asymmetry with respect to the sign of $\Delta$. For the cases $\Omega<\Gamma$ (Fig. 2 (a,b) ) the reason lies mostly in the level renormalization discussed above. This can be deduced from the fact that the curves without real parts (noR) that neglect the level renormalization are actually approximately symmetric. However, for $\Omega>\Gamma$ [Fig. 2 (c)] all numerical approaches give an additional step at $e V_{\text {bias }} / 2 \approx E_{1}+U$ for positive $\Delta=2 \Gamma$, which is absent for negative $\Delta$.

For the case of large $\Omega$ the "molecular" bonding and anti-bonding states provide an appropriate description of the single-electron states of the DQD (see also the comparison in App. B). Because of the large (positive) detuning $\Delta>\Gamma, k_{B} T$ the wave function of the bonding state has much more weight on the right dot (interface to the collector lead) than on the left dot. Correspondlingly, the anti-bonding state has more weight on the left dot (interface to the emitter electrode). This asymmetry in the wave functions leads to an effective asymmetry of coupling of these states to the leads, e.g. for positive $\Delta$, the anti-bonding state is strongly coupled to the emitter, whereas the bonding state is strongly coupled to the collector lead.

The eigenenergies $E_{1,2}$ of the bonding and antibonding state are given by $\mp \sqrt{\Delta^{2}+4 \Omega^{2}} / 2=\mp \sqrt{5} \Gamma$ (see section (II). As $E_{1}=-\sqrt{5} \Gamma$ is the only state with negative energy, it is the overall ground state of the DQD. This means that in equilibrium (zero bias) the bonding state is mainly occupied, whereas all other states have only small occupation probability. The first current step appears at a bias $e V_{\text {bias }} / 2 \sim\left|E_{1}\right| \approx 2.2 \Gamma$ when the DQD can be emptied. At the same bias the anti-bonding state can be occupied by tunneling of an electron from the emitter into the empty DQD. Because of the coupling asymmetry, above a bias $e V_{\text {bias }} / 2 \sim\left|E_{1}\right|$ there is a large change in the average occupation of the DQD states: the bonding state is strongly depleted due to the good coupling to the collector lead, whereas the anti-bonding state is now favorably filled by electrons tunneling into the DQD from the emitter lead. Thus, on the first plateau the DQD is most of the time in the anti-bonding state.

As the bias is further increased, the doubly occupied state comes within energetic range when the bonding state can also be filled in addition to the anti-bonding state. This happens at $e V_{\text {bias }} / 2=E_{1}+U=(-\sqrt{5}+$ $U) \Gamma \approx 7.8 \Gamma$, which is where the second current step sets in. Now, the anti-bonding state loses occupation in favor of the double occupied state and the bonding state. Finally, at even larger bias $e V_{\text {bias }} / 2=E_{2}+U=$ $(\sqrt{5}+10) \Gamma \approx 12.2 \Gamma$, the doubly occupied state can be populated by an electron tunneling in from the emitter, even if the DQD is previously in the bonding state. This leads to the (weak) third current step for the curves corresponding of positive values of bias and $\Delta$. 
In contrast, for negative $\Delta$ and positive bias, because of the reversed spatial asymmetry of bonding and antibonding wave functions, the DQD remains mostly in the bonding state, even though the bias has exceeded $e V_{\text {bias }} / 2 \sim\left|E_{1}\right| \approx 2.2 \Gamma$. The occupation of the antibonding state remains very small in the range $2.2 \Gamma<$ $e V_{\text {bias }} / 2<12.2 \Gamma$. Therefore, the middle current plateau is strongly suppressed and smeared out by the thermal (and linewidth) broadening.

The quantum rate equation results Eqs. (23) correspond to the current values of the first and third current plateau. To capture the middle plateau one would have to account for the possible transitions from the antibonding state to the doubly occupied state in the relevant bias range. This was not done in Ref. 18, consequently the middle plateau is never observed in their Fig. 6 even for the case $\Omega=\Gamma$.

\section{NDC due to decoherence}

Now we focus on the behavior around $e V_{\text {bias }} \approx 2 U$, where the double occupied state enters the window between the left and the right chemical potential. Fig. 11(a) shows that the current peak is significantly larger for $e V_{\text {bias }}<2 U$ (region 1) than for $e V_{\text {bias }}>2 U$ (region 2) for small $\Omega$. Just the opposite holds for large $\Omega$, see Fig.1(c).

This drop of current has been addressed by Djuric et $a l .{ }^{18}$, using the quantum rate equation formalism developed in Ref. 27. They restricted the analysis to a fully symmetric system, i.e. $\Delta=0$, and did not include any real parts. In this case Eqs. (23) provide $I_{1}>I_{2}$ for $\Omega<\sqrt{\Gamma_{L} \Gamma_{R}} / 2$, and $I_{1}<I_{2}$ for $\Omega>\sqrt{\Gamma_{L} \Gamma_{R}} / 2$. Numerically, they observe a smooth interpolation between these plateau values upon variation of $V_{\text {bias }}$, similar to the result of the $1 \mathrm{vN}$ approach without real parts (dotdashed line) in Fig. 2. This gives rise to NDC around $e V_{\text {bias }} \approx 2 U$ for weak inter-dot coupling $\Omega<\sqrt{\Gamma_{L} \Gamma_{R}} / 2$.

While the observed increase of current for $\Omega>$ $\sqrt{\Gamma_{L} \Gamma_{R}} / 2$ can be easily attributed to the opening of a new current channel, the NDC for $\Omega<\sqrt{\Gamma_{L} \Gamma_{R}} / 2$ is less straightforward. In the limit of small $\Omega$, Eqs. (23) read

$$
I_{1} \approx \frac{e}{h} \Omega^{2} L\left(\Delta, \Gamma_{R}\right), \quad I_{2} \approx \frac{e}{h} \Omega^{2} L\left(\Delta, \Gamma_{R}+\Gamma_{L}\right)
$$

with the Lorentzian $L(\Delta, \gamma)=\frac{\gamma}{\Delta^{2}+(\gamma / 2)^{2}}$. This is just the expression from Fermi's golden rule for sequential tunneling between the localized dot states, which is limiting the current for weak coupling, see Ref. 28. The broadening $\gamma$ is given by the dephasing of the coherence between the localized dot states 29 . For the second plateau $I_{2}$ the interdot Coulomb repulsion does not play a role and we get the broadening $\gamma=\Gamma_{R}+\Gamma_{L}$. However, for $e V_{\text {bias }}<2 U$, the left contact cannot add an electron to the system if one electron is already present in the DQD. Thus, the dephasing of the coherent transitions between the dots is only due to $\gamma=\Gamma_{R}$. Therefore, in Fig. 1(a), the current peak (at fixed bias voltage) is higher and narrower (as a function of $\Delta$ ) for $e V_{\text {bias }}<2 U$ (first plateau) in comparison to $e V_{\text {bias }}>2 U$ (second plateau).

As displayed by Fig. 2 the transition between the plateau values for $\Delta=0$ is much more complex than the smooth transition suggested by Ref. 18. Both the $1 \mathrm{vN}$ and the $2 \mathrm{vN}$ approach do not reach a stable value at the first plateau even for $U$ being by far the largest energy. Instead the current drops over the full length of the plateau, giving a much weaker NDC than predicted by Ref. 18. The plateau value $I_{2}$ is indeed reached in region 2 for $V_{\text {bias }} \rightarrow \infty$, but due to the level renormalization effect the transition is much broader than if it was only given by temperature (or even a combination of temperature and line-width broadening $\Gamma$ ). For $\Omega=\sqrt{\Gamma_{L} \Gamma_{R}} / 2$ (Fig. 2(b)) the current does not reach the plateau value $I_{1}$, and at the transition between the two regimes $\left(e V_{\text {bias }} \simeq 2 U=20 \Gamma\right)$ a dip in the current is observed. For $\Omega=2 \Gamma$ (see Fig. 2(c)) where $I_{1}<I_{2}$ and no NDC should occur due to decoherence, we observe that the plateau values are indeed reached in both regimes. While Fig. 2(a) shows very non-monotonous behavior, Fig. 1] shows that the current varies more continously in the $\left(\Delta, V_{\text {bias }}\right)$ plane. This shows that the main difference between our $1 \mathrm{vN}$ approach and the result of Ref. 18 is the level renormalization discussed above resulting in an effective $\Delta_{\text {eff }}$, which is not contained in the quantum rate equation formalism of Ref. 27 .

So far we have only considered a fixed value of the Coulomb repulsion $U=10 \Gamma$. Fig. 4 shows results for different values of $U$ at weak coupling $\Omega=\Gamma / 10$ and zero detuning $\Delta=0$. It should be noted that even for $U$ being by far the dominant energy (e.g. $U=15 \Gamma$ ) no clear plateau value is observed, but instead a slow cross-over between the two regimes. Again, relatively small differences between the $1 \mathrm{vN}$ and $2 \mathrm{vN}$ approach are observed, though in general the $2 \mathrm{vN}$ approach reduces somewhat the level renormalization effect and leads to more plateau-like current-bias characteristic than the $1 \mathrm{vN}$ approach. Here, a particular surprising feature is the fact that a small (unphysical) NDC remains even for $U=0$ in the $1 \mathrm{vN}$ approach (see also the analytical result in App. B] , which is however absent in the $2 \mathrm{vN}$ approach (in full agreement with the transmission result by Green functions).

We conclude that the NDC-scenario due to decoherence outlined in Ref. 18 is strongly modified by the level renormalizations addressed in section IVB. Also note that the high-bias limit $I_{2}$ is only reached for very high bias in the case of weak or intermediate inter-dot coupling $\Omega$ as shown in Fig. 2 .

\section{E. Transport with both spins}

While we restricted ourselves to the case of spinless fermions before, we now take into account both spin directions in both dots, thereby accounting for sixteen different many-particle states. We add an intradot Coulomb 


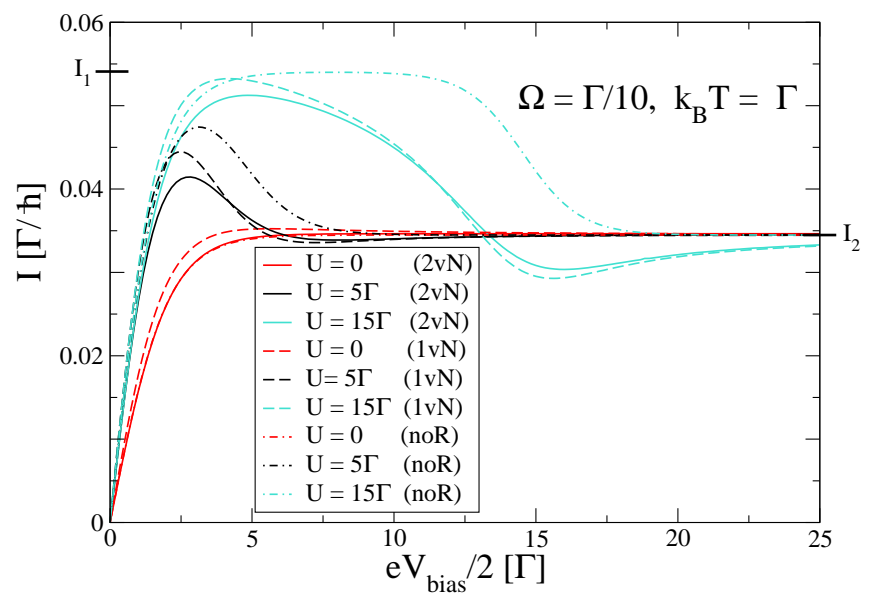

FIG. 4: Results for $\Delta=0, \Omega=\Gamma / 10$, and different values of $U$. All other parameters as in Figs. 112

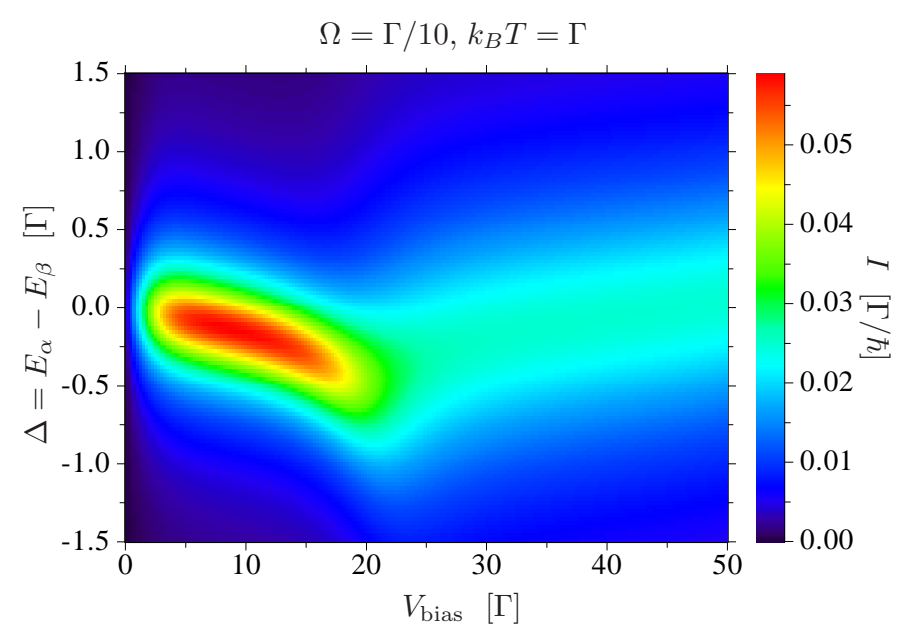

FIG. 5: The current taking into account both spin directions and an intradot Coulomb repulsion of $U_{\text {intra }}=30 \Gamma$ calculated by the $1 \mathrm{vN}$ approach. All other parameters as Fig. 1(a).

repulsion of $U_{\text {intra }}=30 \Gamma$ between the different spin states within each dot, while the interdot Coulomb repulsion $U=10 \Gamma$ is assumed to be independent on the spin direction. The result from the $1 \mathrm{vN}$ approach is shown in Fig. [5] which is qualitatively similar to Fig. 1(a) where spinless electrons were considered. However, quantitatively, the features of level renormalization and NDC due to coherence are enhanced.

\section{DISCUSSION AND SUMMARY}

We have studied transport trough a double quantum dot system and investigated various sources of negative differential conductance (NDC) potentially observable in experiment. Our method reproduces the basic features of Refs. 17, 18, which constitute certain limits of our full numerical approach. In particular we can treat all values of inter-dot coupling and detuning of dot levels within the $1 \mathrm{vN}$ approach. Further effects of line-width broadening due to higher order tunneling events can be taken into account by the $2 \mathrm{vN}$ approach. The NDC due to level renormalization (Sec. IVB) as introduced by Ref. 17 using a strictly sequential tunneling scenario is only quantitatively modified by the higher order tunneling events (even at temperatures comparable to the line-width broadening), thus showing a surprising robustness. The NDC due to decoherence $\frac{18}{}$ (Sec. IVD) is clearly seen in the heights of the current peaks in the bias-detuning plane, see Fig. 1. Nevertheless this effect is strongly masked by the level renormalization effects if a constant detuning $\Delta$ is considered. Both effects show the relevance of a consistent treatment of first-order tunneling terms, which can be achieved by the $1 \mathrm{vN}$ approach discussed here. Comparison with the higher-order $2 \mathrm{vN}$ approach ${ }^{19}$ for a large variety of parameters shows that the validity of these first-order approaches is only restricted to the temperature being larger than the level broadening.

\section{Acknowledgments}

This work was supported by the Swedish Research Council (VR) and the Villum Kann Rasmussen fond.

\section{APPENDIX A: FIRST ORDER VON NEUMANN APPROACH}

We denote the many particle states by $|a\rangle,|b\rangle \ldots$ with energies $E_{a}, E_{b}, \ldots$, respectively, and we use the convention, that the particle number follows the position of the letter in the alphabet, i.e. $|a\rangle$ is an $N$-particle state and $|b\rangle$ is an $N+1$-particle state. The tunnel matrix elements $T_{b a}(k \sigma \ell)$ for a transition from the state $|a\rangle$ to $|b\rangle$ by entering of an electron from the contact $\ell \in\{L, R\}$ with spin $\sigma$ and momentum $k$ can be directly related to the single particle tunneling Hamiltonian as given in Eq. (1), see App. A of Ref. 19. The key quantities are the elements of the reduced density matrix $w_{b^{\prime} b}=$ Trace $\left\{|b\rangle\left\langle b^{\prime}\right| \hat{\rho}\right\}$, where the diagonal elements are the probabilities to find the respective many-particle state and the off-diagonal elements refer to correlations between the many-body states induced via coherent tunneling processes to and from the leads.

The equation of motion for $w_{b^{\prime} b}$ is derived from the von Neumann equation for the density matrix, see Eq. (11) of Ref. 19, and depends on the current amplitudes $\phi_{c b}(k \sigma \ell)=\operatorname{Trace}\left\{|b\rangle \hat{c}_{k \sigma \ell}^{\dagger}\langle c| \hat{\rho}\right\}$. These $\phi_{c b}(k \sigma \ell)$ are themselves determined by an equation of motion, see Eq. (7) of Ref. 19. In the first-order approach, all terms containing both $k$ and $k^{\prime}$ (correlations between two transitions) are neglected and Eq. (10) of Ref. 19 has the solution 


$$
\phi_{c b}(k \sigma \ell)(t)=\frac{1}{\mathrm{i} \hbar} \int_{-\infty}^{t} \mathrm{~d} t^{\prime} \mathrm{e}^{\mathrm{i}\left(E_{b}+E_{k}-E_{c}+\mathrm{i} 0^{+}\right)\left(t-t^{\prime}\right) / \hbar}\left(\sum_{b^{\prime}} T_{c b^{\prime}}(k) w_{b^{\prime} b}\left(t^{\prime}\right) f_{k}-\sum_{c^{\prime}} w_{c c^{\prime}}\left(t^{\prime}\right) T_{c^{\prime} b}(k)\left(1-f_{k}\right)\right)
$$

Now we neglect the time dependence of $w_{b^{\prime} b}\left(t^{\prime}\right)$ in the kernel of the integral (the Markov limit) and set $w_{b^{\prime} b}\left(t^{\prime}\right)=$ $w_{b^{\prime} b}(t)$, which allows us to perform the integral. Inserting this into the equation of motion for $w_{b^{\prime} b}$, we obtain

$$
\begin{aligned}
\mathrm{i} \hbar \frac{\mathrm{d}}{\mathrm{d} t} w_{b b^{\prime}}= & \left(E_{b}-E_{b^{\prime}}\right) w_{b b^{\prime}} \\
& +\sum_{a, k \sigma \ell} T_{b a}(k \sigma \ell) \frac{\sum_{a^{\prime}} w_{a a^{\prime}} T_{b^{\prime} a^{\prime}}^{*}(k \sigma \ell) f_{\ell}\left(E_{k}\right)-\sum_{b^{\prime \prime}} T_{b^{\prime \prime} a}^{*}(k \sigma \ell) w_{b^{\prime \prime} b^{\prime}}\left(1-f_{\ell}\left(E_{k}\right)\right)}{E_{k}-E_{b^{\prime}}+E_{a}-\mathrm{i} 0^{+}} \\
& -\sum_{a, k \sigma \ell} \frac{\sum_{a^{\prime}} T_{b a^{\prime}}(k \sigma \ell) w_{a^{\prime} a} f_{\ell}\left(E_{k}\right)-\sum_{b^{\prime \prime}} w_{b b^{\prime \prime}} T_{b^{\prime \prime} a}(k \sigma \ell)\left(1-f_{\ell}\left(E_{k}\right)\right)}{E_{k}-E_{b}+E_{a}+\mathrm{i} 0^{+}} T_{b^{\prime} a}^{*}(k \sigma \ell) \\
& +\sum_{c, k \sigma \ell} T_{c b}^{*}(k \sigma \ell) \frac{\sum_{b^{\prime \prime}} T_{c b^{\prime \prime}}(k \sigma \ell) w_{b^{\prime \prime} b^{\prime}} f_{\ell}\left(E_{k}\right)-\sum_{c^{\prime}} w_{c c^{\prime}} T_{c^{\prime} b^{\prime}}(k \sigma \ell)\left(1-f_{\ell}\left(E_{k}\right)\right)}{E_{k}-E_{c}+E_{b^{\prime}}+\mathrm{i} 0^{+}} \\
& -\sum_{c, k \sigma \ell} \frac{\sum_{b^{\prime \prime}} w_{b b^{\prime \prime}} T_{c b^{\prime \prime}}^{*}(k \sigma \ell) f_{\ell}\left(E_{k}\right)-\sum_{c^{\prime}} T_{c^{\prime} b}^{*}(k \sigma \ell) w_{c^{\prime} c}\left(1-f_{\ell}\left(E_{k}\right)\right)}{E_{k}-E_{c}+E_{b}-\mathrm{i} 0^{+}} T_{c b^{\prime}}(k \sigma \ell) .
\end{aligned}
$$

The current from the left lead into the system is given by

$$
J_{L}=-\frac{2}{\hbar} \Im\left\{\sum_{k \sigma, c b} \frac{T_{c b}^{*}(k \sigma L)}{E_{k}-E_{c}+E_{b}+\mathrm{i} 0^{+}}\left(\sum_{b^{\prime}} T_{c b^{\prime}}(k \sigma L) w_{b^{\prime} b} f_{L}\left(E_{k}\right)-\sum_{c^{\prime}} w_{c c^{\prime}} T_{c^{\prime} b}(k \sigma L)\left(1-f_{L}\left(E_{k}\right)\right)\right)\right\} .
$$

If we restrict to diagonal elements $P_{b}=w_{b b}$, these equations reduce to the standard master equation ${ }^{30}$ formulated in a many particle basis $31,32,33$.

The Redfield kinetics ${ }^{34}$ has been recently used to derive a similar set of equations ${ }^{35}$. We can recover these equations, if we approximate $w_{b^{\prime} b}\left(t^{\prime}\right)=$ $w_{b^{\prime} b}(t) \mathrm{e}^{-\mathrm{i}\left(E_{b^{\prime}}-E_{b}\right)\left(t^{\prime}-t\right) / \hbar}$ in Eq. (A1). In this case the integrations can be performed as well, but we obtain slightly different denominators for the nondiagonal elements in Eq. (A2). While the approximation $w_{b^{\prime} b}\left(t^{\prime}\right)=$ $w_{b^{\prime} b}(t)$ becomes exact in the stationary state, which we consider here, the behavior $w_{b^{\prime} b}\left(t^{\prime}\right) \propto \mathrm{e}^{-\mathrm{i}\left(E_{b^{\prime}}-E_{b}\right) t^{\prime} / \hbar}$ is suggested by the linear term in the equation of motion. $\underline{42}^{2}$ At the moment, we have no direct indication, which concept is more appropriate. However, we did only find minor numerical differences and the qualitative features are identical for all issues discussed in this article. In particular, both approaches can yield negative probabilities $w_{b b}$, a well-known problem of Redfield kinetics ${ }^{36}$.

\section{APPENDIX B: COMPARISON OF DIFFERENT APPROACHES IN THE NONINTERACTING LIMIT}

Now we consider the double dot model without spin in the noninteracting limit $U=0$, and set $E_{\alpha}=E_{\beta}=0$, $\Gamma_{L}=\Gamma_{R}=\Gamma / 2$ as well as $W \rightarrow \infty$. In this case we can solve most approaches analytically, allowing for a better understanding of the structure of the various approaches.

\section{Transmission formalism and $2 \mathrm{vN}$ approach}

As a bench mark, for the noninteracting case the current can be evaluated exactly via the transmission formalism (see e.g. Ref. 37)

$$
I=\frac{1}{2 \pi \hbar} \int \mathrm{d} E T(E)\left[f_{L}(E)-f_{R}(E)\right]
$$

with

$$
T(E)=\frac{\Gamma^{2} \Omega^{2}}{4\left[(E-\Omega)^{2}+\Gamma^{2} / 16\right]\left[(E+\Omega)^{2}+\Gamma^{2} / 16\right]}
$$

where the wide band limit is applied. We obtained numerically the same result from the $2 \mathrm{vN}$ approach for all parameters checked.

If $\Gamma \ll k_{B} T$, we may replace the peaks in the transmission function by $\delta$-functions which provides us with

$$
T(E) \approx \begin{cases}\frac{\pi \Gamma}{4}[\delta(E-\Omega)+\delta(E+\Omega)] & \text { for } \Gamma \ll \Omega \\ \frac{2 \pi \Omega^{2} \Gamma}{4 \Omega^{2}+\Gamma^{2} / 4} \delta(E) & \text { for } \Omega \ll \Gamma\end{cases}
$$

The prefactor for $\Omega \ll \Gamma$ is chosen such, that the integral over $E$ agrees with the full transmission function for all $\Omega, \Gamma$. 


\section{Master equation}

The master equation in the many-particle states ${ }^{31,32}$ can be derived by setting $w_{b b^{\prime}}=P_{b} \delta_{b b^{\prime}}$ in the $1 \mathrm{vN}$ approach, resulting in the current

$$
I_{\text {master }}=\frac{\Gamma}{8 \hbar}\left[f_{L}(\Omega)-f_{R}(\Omega)+f_{L}(-\Omega)-f_{R}(-\Omega)\right]
$$

which exactly equals the bench mark (B1) in the limit $\Gamma \ll k_{B} T, \Omega$, see Eq. (B3).

\section{Quantum rate equation}

Going beyond the master equation, correlations between different states can be taken into account. The dephasing of these correlations is frequently treated in a Lindblad form ${ }^{36.38}$, see, e.g., Refs. 22, 23, 24, 27. They can be derived in different ways and the name "quantum rate equation" is frequently used. Formulated in a basis of the localized states, see e.g. Ref. 22 or Eq. (36) of Ref. 27, we find the result

$$
I_{\text {quantum rate }}=\frac{\Gamma}{\hbar} \frac{\Omega^{2}}{\Gamma^{2} / 4+4 \Omega^{2}}\left(f_{L}(0)-f_{R}(0)\right),
$$

which matches perfectly the bench mark result in the limit $\Omega \ll \Gamma \ll k_{B} T$, see Eq. (B3). In addition, it is correct in the high-bias limit, $\mu_{L},\left(-\mu_{R}\right) \gg \Omega, \Gamma$, as proven by Gurvitz and Prager ${ }^{22}$

\section{1vN approach}

Finally the $1 \mathrm{vN}$ approach provides

$$
\begin{aligned}
I_{1 \mathrm{vN}}= & \frac{1}{\hbar} \frac{\Gamma \Omega^{2}}{\Gamma^{2} / 4+4 \Omega^{2}} \frac{\left[f_{L}(\Omega)-f_{R}(\Omega)+f_{L}(-\Omega)-f_{R}(-\Omega)\right]}{2} \\
& +\frac{A}{8 \hbar} \frac{\Omega \Gamma^{2}}{\Gamma^{2} / 4+4 \Omega^{2}}
\end{aligned}
$$

where

$$
A=\frac{1}{\pi} \int_{-\infty}^{\infty} \mathrm{d} E\left[f_{L}(E)-f_{R}(E)\right] \mathcal{P}\left\{\frac{1}{E+\Omega}-\frac{1}{E-\Omega}\right\}
$$

is a small contribution from the real parts. If the real parts are neglected $(A=0)$, the result matches the bench mark result as long as $k_{B} T>\Gamma$. This indicates that the inclusion of the real parts may not be appropriate in the noninteracting limit for a first order approach in the coupling $\Gamma$, see also the little peak at $e V_{\text {bias }}=2 \Gamma$ for $U=0$ in Fig. 4. However, the comparison with the $2 \mathrm{vN}$ approach (which does not display the spurious peak) indicates that the real parts cover the essential physics in the interacting case. It is interesting to note, that the Redfield kinetics provides exactly the same analytical result (B6), so that both approaches exhibit the same problem in the noninteracting case.

\section{APPENDIX C: COMPARISON WITH}

\section{REAL-TIME DIAGRAMMATIC APPROACH}

The $2 \mathrm{vN}$ approach allows for an analytic solution for the single dot model with spin (the Anderson model) with infinite Coulomb repulsion. We consider a spindegenerate level with $E_{\uparrow}=E_{\downarrow}=E_{d}$. Analogously to section III of Ref. 19, we define

$$
\begin{aligned}
B_{\sigma ; 0}^{\ell}(E) & =\sum_{k} \delta\left(E-E_{k}\right) T_{\ell}(k) \phi_{\sigma, 0}(k \sigma \ell) \\
B_{\sigma ; 0}(E) & =B_{\sigma ; 0}^{L}(E)+B_{\sigma ; 0}^{R}(E) \\
\Gamma_{\ell}(E) & =2 \pi \sum_{k} \delta\left(E-E_{k}\right) T_{\ell}^{2}(k) \\
\Gamma(E) & =\Gamma_{L}(E)+\Gamma_{R}(E)
\end{aligned}
$$

where we assume that the couplings to the contacts $T_{\uparrow 0}(k \uparrow \ell)=T_{\downarrow 0}(k \downarrow \ell)=T_{\ell}(k)$ do not depend on spin. Then we obtain

$$
\begin{gathered}
\mathrm{i} \hbar \frac{\mathrm{d}}{\mathrm{d} t} B_{\sigma ; 0}^{\ell}(E)=\left(E_{d}-E+\Sigma(E)+\Sigma^{f}(E)\right) B_{\sigma ; 0}^{\ell}(E) \\
+\frac{\Gamma_{\ell}(E)}{2 \pi}\left[w_{0 ; 0} f_{\ell}(E)-w_{\sigma ; \sigma}\left(1-f_{\ell}(E)\right)\right] \\
-\frac{\Gamma_{\ell}(E)}{2 \pi} \int \mathrm{d} E^{\prime} \frac{B_{\sigma, 0}^{*}\left(E^{\prime}\right)+f_{\ell}(E) B_{\bar{\sigma}, 0}^{*}\left(E^{\prime}\right)}{E-E^{\prime}+\mathrm{i} 0^{+}}
\end{gathered}
$$

where $\bar{\sigma}$ denotes the spin opposite to $\sigma$ and

$$
\begin{aligned}
\Sigma\left(E_{k}\right) & =\sum_{k^{\prime} \ell} \frac{T_{\ell^{\prime}}\left(k^{\prime}\right)^{2}}{E_{k}-E_{k^{\prime}}+\mathrm{i} 0^{+}} \\
\Sigma^{f}\left(E_{k}\right) & =\sum_{k^{\prime} \ell} \frac{f_{k^{\prime}} T_{\ell^{\prime}}\left(k^{\prime}\right)^{2}}{E_{k}-E_{k^{\prime}}+\mathrm{i} 0^{+}}
\end{aligned}
$$

as well as

$$
\begin{aligned}
& \hbar \frac{\mathrm{d}}{\mathrm{d} t} w_{\sigma, \sigma}=-2 \Im\left\{\int \mathrm{d} E B_{\sigma, 0}(E)\right\} \\
& \hbar \frac{\mathrm{d}}{\mathrm{d} t} w_{0,0}=2 \Im\left\{\int \mathrm{d} E B_{\uparrow, 0}(E)+B_{\downarrow, 0}(E)\right\}
\end{aligned}
$$

With the Ansatz $B_{\uparrow ; 0}=B_{\downarrow ; 0}=B(E)$ and $w_{\uparrow, \uparrow}=w_{\downarrow ; \downarrow}$ we have the stationary solution 


$$
\begin{aligned}
\frac{\Gamma_{L}\left(1+f_{L}(E)\right)+\Gamma_{R}\left(1+f_{R}(E)\right)}{2 \pi} \int \mathrm{d} E^{\prime} \frac{B^{*}\left(E^{\prime}\right)}{E-E^{\prime}+\mathrm{i} 0^{+}}=\left(E_{d}-E+\Sigma(E)+\Sigma^{f}(E)\right) B(E) \\
+\frac{w_{0,0}\left[\Gamma_{L}(E) f_{L}(E)+\Gamma_{R}(E) f_{R}(E)\right]}{2 \pi}-\frac{w_{\sigma, \sigma}\left[\Gamma_{L}(E)\left(1-f_{L}(E)\right)+\Gamma_{R}(E)\left(1-f_{R}(E)\right)\right]}{2 \pi}
\end{aligned}
$$

As

$$
\Im\left\{\Sigma(E)+\Sigma^{f}(E)\right\}=-\left[\Gamma_{L}(E)\left(1+f_{L}(E)\right)+\Gamma_{R}(E)\left(1+f_{R}(E)\right)\right] / 2
$$

we find that there is a solution $B(E)$ which is purely real (like in the spinless level case). Inserting into Eq. (C1) gives the stationary state

$$
\begin{aligned}
\left(E_{d}-E+\Sigma(E)+\Sigma^{f}(E)\right) B_{\sigma ; 0}^{L}(E)=\frac{\Gamma_{L}\left[1+f_{L}(E)\right]\left(E_{d}-E\right.}{\Gamma_{L}(E)\left(1+f_{L}(E)\right)}+ & \Gamma_{R}(E)\left(1+\Sigma_{R}^{f}(E)\right) B(E) \\
& +\frac{\Gamma_{L} \Gamma_{R}\left(f_{R}(E)-f_{L}(E)\right)}{2 \pi\left[\Gamma_{L}(E)\left(1+f_{L}(E)\right)+\Gamma_{R}(E)\left(1+f_{R}(E)\right)\right]}
\end{aligned}
$$

where we used $w_{0 ; 0}+2 w_{\sigma, \sigma}=1$. With Eq. (C4) we have

$$
\Im\left\{B_{\sigma ; 0}^{L}(E)\right\}=\frac{\Gamma_{L}(E) \Gamma_{R}(E)\left(f_{R}(E)-f_{L}(E)\right)}{4 \pi\left|E_{d}-E+\Sigma(E)+\Sigma^{f}(E)\right|^{2}}
$$

Finally the particle current (including both spin directions) is given by:

$$
J_{L}=\frac{1}{\hbar} \int \mathrm{d} E \frac{\Gamma_{L}(E) \Gamma_{R}(E)\left(f_{L}(E)-f_{R}(E)\right)}{\pi\left|E_{d}-E+\Sigma(E)+\Sigma^{f}(E)\right|^{2}}
$$

This result fully agrees with the result from real-time perturbation theory in the so-called resonant tunneling approximation (containing a resummation of diagrams beyond second order perturbation theory), see Eq. (4.61) of Ref. 39. This indicates that the $2 \mathrm{vN}$ approach contains an equivalent set of higher than second order tunneling processes due to the self-consistency in the equation of motion for $\phi_{c b}(k)$.
* Now at Mads Clausen Institute, University of Southern Denmark, Grundtvigs Allé 150, 6400 Sønderborg, Denmark.

1 M. A. Kastner, Rev. Mod. Phys. 64, 849 (1992).

2 M. Pustilnik and L. Glazman, J. Phys.: Condens. Matter 16, R513 (2004).

3 W. G. v. Wiel, S. D. Franceschi, J. M. Elzerman, T. Fujisawa, S. Tarucha, and L. P. Kouwenhoven, Rev. Mod. Phys. 75, 1 (2003).

4 T. Hatano, M. Stopa, T. Yamaguchi, T. Ota, K. Yamada, and S. Tarucha, Phys. Rev. Lett. 93, 066806 (2004).

${ }^{5}$ L. DiCarlo, H. J. Lynch, A. C. Johnson, L. I. Childress, K. Crockett, C. M. Marcus, M. P. Hanson, and A. C. Gossard, Phys. Rev. Lett. 92, 226801 (2004).

6 A. K. Huttel, S. Ludwig, H. Lorenz, K. Eberl, and J. P. Kotthaus, Phys. Rev. B 72, 081310 (2005).

7 C. Fasth, A. Fuhrer, M. T. Björk, and L. Samuelson, Nano Letters 5, 1487 (2005).

8 M. Sigrist, T. Ihn, K. Ensslin, D. Loss, M. Reinwald, and W. Wegscheider, Phys. Rev. Lett. 96, 036804 (2006).

9 T. Brandes, phys. status solidi (b) 243, 2293 (2006).

10 A. Fuhrer, L. E. Fröberg, J. N. Pedersen, M. W. Larsson, A. Wacker, M.-E. Pistol, and L. Samuelson, Nano Letters 7, 243 (2007).

11 J. Weis, R. J. Haug, K. v. Klitzing, and K. Ploog, Phys. Rev. Lett. 71, 4019 (1993).
12 D. Weinmann, W. Häusler, and B. Kramer, Phys. Rev. Lett. 74, 984 (1995).

13 M. H. Hettler, H. Schoeller, and W. Wenzel, Europhys. Lett. 57, 571 (2002).

14 M. C. Rogge, F. Cavaliere, M. Sassetti, R. J. Haug, and B. Kramer, New Journal of Physics 8, 298 (2006).

15 A. Nauen, F. Hohls, N. Maire, K. Pierz, and R. J. Haug, Phys. Rev. B 70, 033305 (2004).

16 J. Aghassi, A. Thielmann, M. Hettler, and G. Schön, Phys. Rev. B 73, 195323 (2006).

17 B. Wunsch, M. Braun, J. König, and D. Pfannkuche, Phys. Rev. B 72, 205319 (2005).

18 I. Djuric, B. Dong, and H. L. Cui, J. Appl. Phys. 99, 063710 (2006).

19 J. N. Pedersen and A. Wacker, Phys. Rev. B 72, 195330 (2005).

20 B. Muralidharan, A. W. Ghosh, and S. Datta, Phys. Rev. B 73, 155410 (2006).

21 B. R. Bulka and T. Kostyrko, Phys. Rev. B 70, 205333 (2004).

22 S. A. Gurvitz and Y. S. Prager, Phys. Rev. B 53, 15932 (1996).

23 T. H. Stoof and Y. V. Nazarov, Phys. Rev. B 53, 1050 (1996).

24 B. Ellatari and S. Gurvitz, Phys. Lett. A 292, 289 (2002).

25 J. König, H. Schoeller, and G. Schön, Phys. Rev. Lett. 76, 
1715 (1996).

26 J. König, J. Schmid, H. Schoeller, and G. Schön, Phys. Rev. B 54, 16820 (1996).

27 B. Dong, H. L. Cui, and X. L. Lei, Phys. Rev. B 69, 035324 (2004).

${ }^{28}$ H. Sprekeler, G. Kießlich, A. Wacker, and E. Schöll, Phys. Rev. B 69, 125328 (2004).

29 G. Kießlich, P. Samuelsson, A. Wacker, and E. Schöll, Phys. Rev. B 73, 033312 (2006).

${ }^{30}$ C. W. J. Beenakker, Phys. Rev. B 44, 1646 (1991).

31 J. M. Kinaret, Y. Meir, N. S. Wingreen, P. A. Lee, and X. Wen, Phys. Rev. B 46, 4681 (1992).

32 G. Cheng, G. Klimeck, S. Datta, G. Chen, and W. A. Goddard, Phys. Rev. B 50, 8035 (1994).

33 D. Pfannkuche and S. E. Ulloa, Phys. Rev. Lett. 74, 1194 (1995).

34 A. G. Redfield, IBM Journal of Research and Development 1, 19 (1957).

35 U. Harbola, M. Esposito, and S. Mukamel, Phys. Rev. B 74, 235309 (2006).
36 U. Weiss, Quantum dissipative systems (World Scientific, London, 1999).

37 S. Datta, Electronic Transport in Mesoscopic Systems (Cambridge University Press, Cambridge, 1995).

38 G. Lindblad, Commun. math. Phys. 48, 119 (1976).

39 J. König, Ph.D. thesis, University Karlsruhe (1998), URL http://www-tfp.physik.uni-karlsruhe.de/Publications/Pub1998/

40 The results in Ref. 16 were derived for a double dot including spin, but the conclusion can be transferred to the spin-less case.

41 A constant $\Delta$ can be realized by minimizing the polarization of the dot levels from the leads (vanishing $\lambda$ ) or by compensating with appropriate gate voltages proportional to $V_{\text {bias. }}$.

${ }^{42}$ For the $2 \mathrm{vN}$-approach, the same ambiguity in closing the equations arises. In this case, the analytical result for the single level problem only matches the Green's function result, if the time dependence is neglected, which is the approximation scheme used in Ref. 19. 